Please send trade news information and illustrations to Arveen Bajaj at the $B D J$, Nature Publishing Group, The Macmillan Building, 4-6 Crinan Street, London N1 9XW.

Trade news is provided as a service to readers using text and images from the manufacturer, supplier or distributor and does not imply endorsement by the $B D J$. Normal and prudent research should be exercised before purchase or use of any product mentioned.

\section{Strong and flexibile}

ParaPost Fiber Lux is the alternative to metal posts for an aesthetic result. It is made of translucent fibre resin material, which reflects the natural hues of a tooth and eliminates shadows through composite restorations and at the gingival margin.

The high percentage of uni-directional fibres give strength and flexibility and the light transmitting material of ParaPost Fiber Lux allows greater control of set times with the added convenience of being able to use self, dual or light-cured materials.

It is available in six sizes which minimises the amount of dentine to be removed and is colour coded for easy identification and compatibility with ParaPost drills.

Reader response number 51

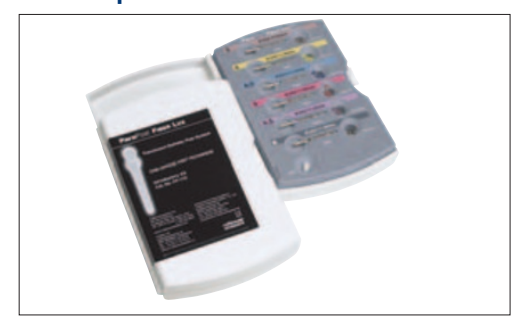

\section{Rapid healing}

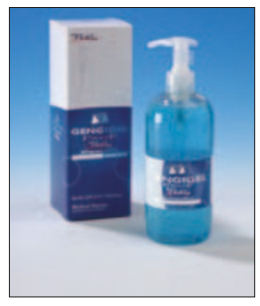

New Gengigel Professional from Oraldent, can promote rapid healing after both surgical and non-surgical procedures. The product is intended for inpractice use where rapid healing is required after surgical intervention, implants, tooth extractions and root scaling.

Gengigel Professional fluid is also beneficial in the treatment of inflammatory conditions such as gingivitis, gingival bleeding and gingival retraction.

The application of hyaluronan, the principal active ingredient of Gengigel, to surgically traumatised gingival and periodontal tissue inhibits inflammation and accelerates tissue repair.

Reader response number 52

\title{
New endodontic micromotor
}

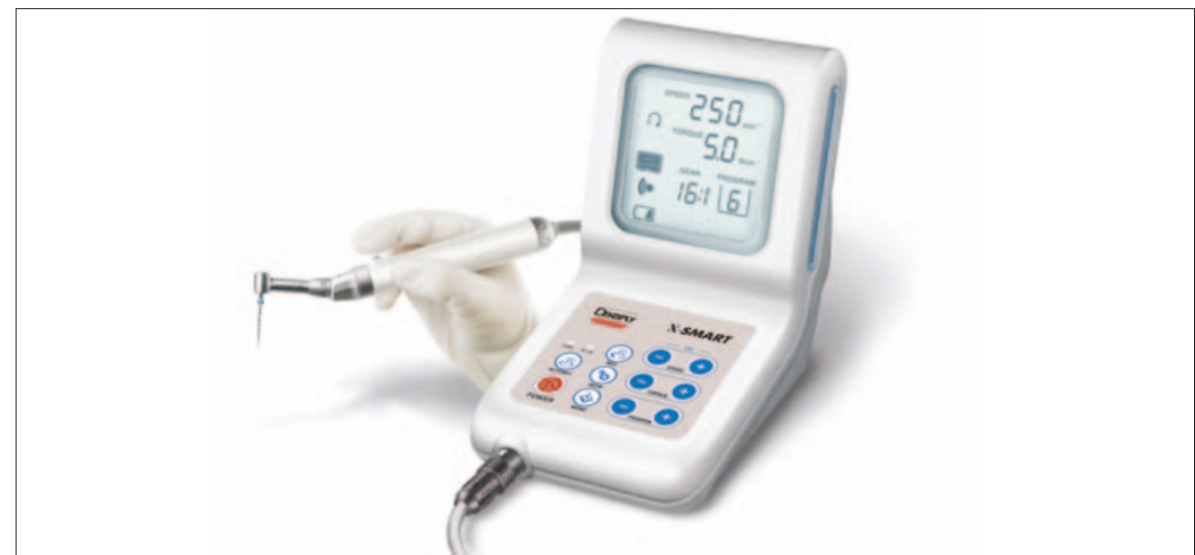

Dentsply Maillefer has announced the launch of the X-Smart, the new endodontic micromotor designed specifically to drive NiTi rotary instruments.

The X-Smart is a simple, compact and lightweight unit adapted to the needs of endodontic practice. The control panel of the X-Smart fits easily into the palm of the hand, yet contains all of the elements the clinician would expect from a motor that is considerably larger.

The motor has three different autoreverse modes to choose from compared to just one that most other motors offer which can reduces the risk of file separation during treatment. The real-time read of the load being applied to the instrument is displayed in the form of a graduated bar

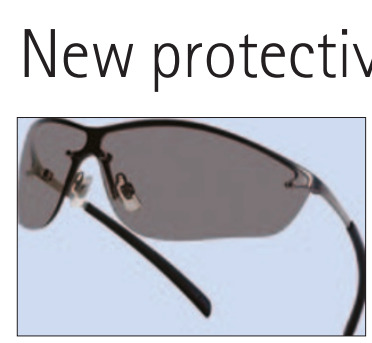

ctive

\section{eyewear range}

The new 2005 protective eyewear range from Cardozo offers a choice of frame for every member of the dental team as well as every patient.

The Pro Specs range has now been enlarged to incorporate the ISO3Z range of frames, which have twin lenses in a wraparound frame. The lenses have been specially treated for maximum scratch resistance and are available in both an adult and junior frame size. The new Viper frame gives panoramic vision with its modern frameless design. It also has an ultralight, adjustable frame for optimum comfort and is available with either clear or tinted lenses.

For a modern, professional appearance, the new Blade frames have a high specification, lightweight metal frame with optically correct wraparound lenses. They are available with a titanium frame colour and choice of either clear or tinted lenses.

Reader response number 53 


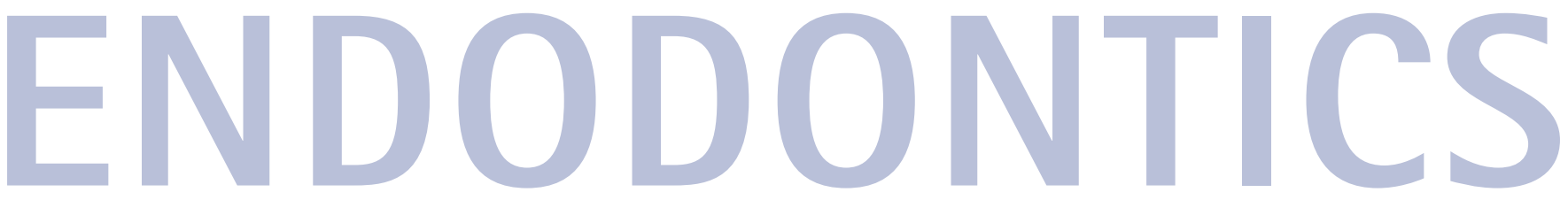

\section{Torque control}

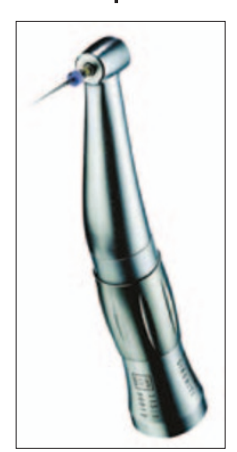

The Sironiti and Sironiti Air root canal handpieces from Sirona can be fitted to any electric micromotor.

The Sironiti Air has a speed reduction of $66: 1$, and if used with an air motor, will produce the optimum speed for endodontic treatment. By turning the pre-select ring for torque control on either one of the two handpieces available, they are ready for use.

There are five torque settings on each handpiece to ensure the correct selection of the recommended torque level for the file system in use.

Safe and effective root canal treatment is assured by the unique 'reto-rotation' function to prevent the file becoming jammed in the root canal if excessive force is applied, fully automatically. Both handpieces may be sterilised in an autoclave at temperatures up to $135^{\circ} \mathrm{C}$. For more details please contact Sirona Dental Systems.

Reader response number 54

\section{Easier location}

C+ Files from Dentsply Maillefer can be used for initial instrumentation during root canal treatment. They offer easier location of canal opening, better negotiation of calcification and better tactile feel.

Markings on the instruments make it easier to determine working length when working with X-rays, while the pyramid shaped tip allows easier insertion during catheterization.

The square cross section provides consistent cutting sensation across the whole range of instruments, thus increasing safety and the choice of three lengths allows optimal efficiency in all clinical access. Reader response number 56

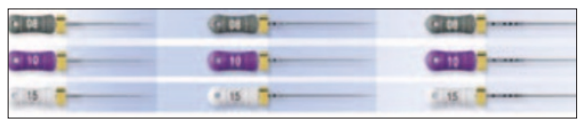

\section{Three-dimensional system}

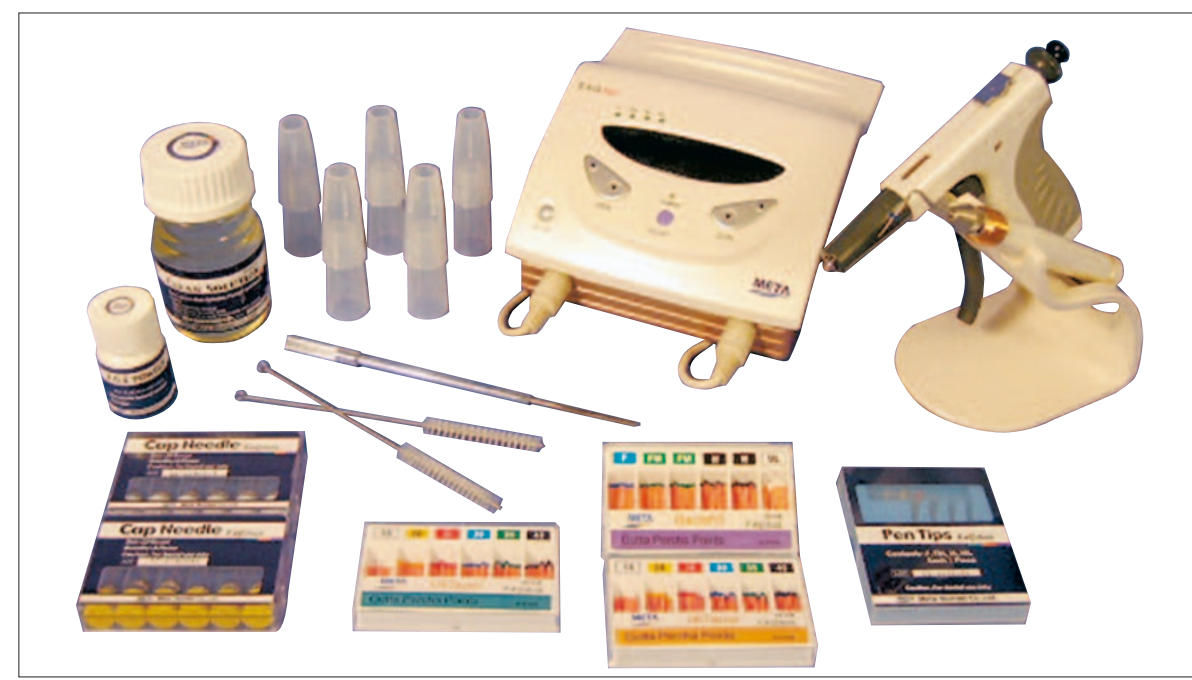

E \& Q Plus is a unique, three-dimensional root canal filling system manufactured by Meta Dental Company and is available exclusively in the UK through Red Apple Dental Supplies.

The E\&Q Plus is two systems in one an automatic heat carrier and gutta percha gun in one compact unit for convenient, fast and easy filling of root canals.

When filling root canals, the dental professional can choose from either vertical compaction of warm gutta percha, heat softened gutta percha injection, or a combination of both techniques.

Condensable gutta percha creates a compact and dense filling and the prod-

\section{Fast replacement}

Filpost pulpal posts are engineered to provide maximum advantages without compromising safety, dentine integrity, retention, passivity and ease of placement. Unlike other post systems, the product can be customised to the canal requirements and two Filposts can be placed in molars for added security.

They are made from pure (99.8\%) Titanium for biocompatibility, have fast, easy replacement, can be bent and customised to fit the canal and have a patented locking system for maximum retention.

There is minimal removal of healthy tooth structure, they are compatible with all dental materials have a passive fit, to minimise fracture potential and as there are only two sizes in the system they can save space and cost. The product is available from Filhol Dental.

Reader response number 57

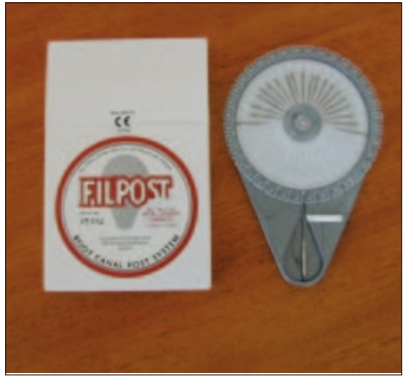




\section{Endodontic re-treatment procedures}

The WAMkey is a solution to endodontic re-treatment procedures that can involve the removal of a crown or the need to drill through the occlusal surface of the crown to access the root canal system.

A small opening in the buccal or lingual aspect of the tooth at a point just between the superior surface of the underlying core and the inferior surface of the underside of the crown is made with a cylindrical diamond to enter the crown and then this hole is extended into the middle of the core. A transmetal bur is used for metallic crowns. WAMkey is then used in this "pilot" hole in a gentle rotation movement on the shaft of the key and working in the axis of the crown, removes it without applying any undue force to tooth or root structure.

If the smaller key is ineffective then WAMkeys 2 and 3 can be used. It can also be used in the removal of bridgework by working alternatively on each abutment. The technique leaves a crown that can be reused after repair or used as a very adequate temporary. The product is available from the Dentsply Maillefer family of endodontic products.

Reader response number 58

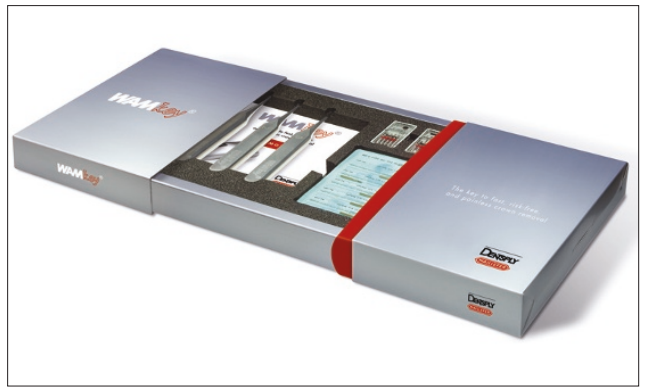

\section{Electromagnetic impulses}

Endox from DPS is a hi-tech piece of equipment that works by emitting a calibrated electromagnetic impulse which reduces bacteria present in the root canal through the electrode that is placed into the dental canal. At the same time it reduces the bacterial load beyond the tooth to adjacent tissue.

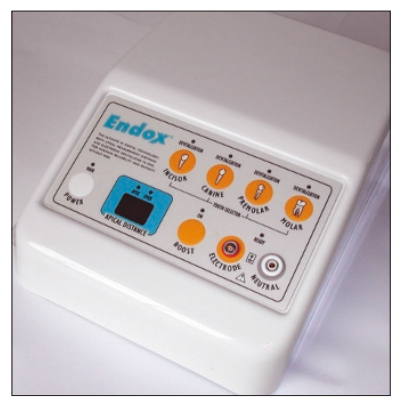

Endox removes pulpal tissue through vaporisation in the canals, lateral canals and dentinal tubules. Bacteria are reduced significantly thus relieving any pain experienced by the patient which can lessen the possibility of the canal becoming re-infected and usually allows complete obturation in the same visit.

Endox prevents the risks that can occur using traditional methods of instrumentation during endodontic treatments by eliminating the creation of false canals, ledges and transporations.

Reader response number 59

\section{Swiss precision}

FKG Endodontic instruments include products such as the Sensipast and Ergoflex files and the Race reamer. The Sensipast paste filler has spirals that automatically disengage when any abnormal pressure is exerted on them to avoid periapical penetration.

Ergoflex $\mathrm{H}$ and $\mathrm{K}$ files have a progressive cutting edge and an ergonomic handle for restricting rotation. This reduces the possibility of introducing them to a greater depth than desired to avoid apex perforation. The stem has a constant diameter for maximum flexibility and has a rounded tip.

Race reamers have alternate cutting edges to eliminate screwing and blocking and the sharp edges give optimum cutting efficiency. In addition the combination of the triangular section and the alternating edges ensures efficient evacuation of chips and debris. The safety tip enables sure guidance and centering of the instrument while the flexibility of the NiTi respects the trajectory of the original canal. The race reamers are available in 2, 4, 6 and 10\% tapers.

All FKG instruments are colour coded to ISO specification and are can be sterilised to $200^{\circ} \mathrm{C}$.

FKG also produces a wide range of endodontic accessories such as stop dispensers, stop cartridges, rubber stops and endo boxes and instruments are manufactured from surgical grade stainless steel with Swiss precision and quality. They are available in the UK through Red Apple Dental Supplies.

Reader response number 60

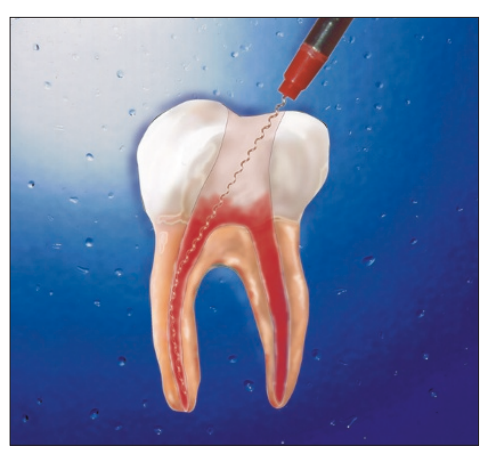

